\title{
Audiological profile in patients with hypothyroidism and hyperthyroidism
}

\begin{abstract}
Aim of the study: Thyroid hormone is important for the normal function of the auditory system. The aim and objective of the study were to investigate the audiological profile in hypothyroidism (group 1) and hyperthyroidism (group 2), to compare the audiological profile between the hypothyroidism and hyperthyroidism.

Material and methods: There with 30 subjects in control group (CG) and 42 subjects (22-hypothyroidism and 20-hyperthyroidism) in study group between age ranges 18-45years. Impedance audiometry, acoustic reflex, OAE, PTA, extended high frequency audiometry and $\mathrm{ABR}$ were performed on all subjects.

Results: Subjects had type-A tympanogram were enrolled in the study. Acoustic reflex thresholds of group 1 and group 2 were elevated both ipsilaterally and contralaterally as compared to CG. In otoacoustic emission, TEOAEs showed decreased SNR in group 1 and group 2 as compared to $\mathrm{CG}$ in both ears. However no significant difference was observed between two study groups viz. group 1 and 2 . Whereas significant difference $(\mathrm{p}<0.05)$ was observed in DPOAEs among CG, group 1 , and $2.77 .3 \%$ of the subjects in group 1 and $84.6 \%$ of the subjects group 2 had minimal hearing loss. Extended high frequency thresholds showed elevated thresholds in group 2 and 3 as compared to group 1. Highly significant $(<0.01)$ difference was observed for absolute latency of wave I and a significant $(p<0.05)$ for waves III and V between CG and group 1 in both ears. No significant $(p>0.05)$ difference was found for interpeak latency between waves I-III, I-V and III-V in either ears in either group.
\end{abstract}

Conclusion: Both hypothyroidism and hyperthyroidism have auditory impairment with minimal degree and it mainly affects cochlea not the external and middle ear. In hypothyroidism cases auditory brainstem is involved. Auditory impairment is more in hypothyroidism as compared to hyperthyroidism.

Keywords: hypothyroidism, hyperthyroidism, puretone audiometry, otoacoustic emissions and abr
Volume 6 Issue 2 - 2017

\author{
Sanjay K Munjal, Rawish Kumar, Sanjay \\ K Munjal, Banumathy N, Sanjay Bhadada, \\ Naresh K Panda \\ Department of Otolaryngology Postgraduate Institute of \\ Medical Education and Research, India
}

Correspondence: Sanjay K Munjal Speech and Hearing Unit Department of Otolaryngology PGIMER Chandigarh, India, Tel +91-9815653262, Email sanjaymunjal I@hotmail.com

Received: December 13, 2016 | Published: February 24, 2017
Abbreviations: PTA, puretone audiometry; OAE, otoacoustic emissions; TEOAE, transient evoked otoacoustic emissions; DPOAE, distortion product otoacoustic emissions; SNR, signal to noise ratio; ART, acoustic reflex testing; ABR, auditory brainstem evoked response

\section{Introduction}

The thyroid hormone is responsible for regulating the metabolic rate of every cell in the body. The action of thyroid hormone is very important in many developmental processes such as body growth and central nervous system maturation. When the thyroid gland is malfunctioning, it can affect anysystem of the body including auditory system. ${ }^{1}$ Thyroid disorder is a general term representing several different diseases involving the thyroid gland and increase or decrease in thyroid hormone. The two major categories in thyroid disorders are, hyperthyroidism (thyroid overactivity) and hypothyroidism (thyroid underactivity), depending on whether serum thyroid hormone levels (T4=Thyroxin and $\mathrm{T} 3=$ Triiodothyronin) are increased or decreased, respectively. Thyroid hormone disorder may be both congenital as well as acquired. According to a projection from several studies on thyroid diseases, it has been estimated that about 42 million people in India suffer from various kind of thyroid diseases. ${ }^{2}$

Hearing loss in patients having hypothyroidism and hyperthyroidism has been reported in literatures. Various researchers reported that the auditory function is particularly sensitive to the effects of thyroid hormone, which is required for the complex development and physiology of the cochlea. In humans, auditory dysfunction is reported frequently in relation to hypothyroidism, with sensorineural hearing impairment ranging from mild to severe degree. ${ }^{3-5}$ Bruschini et al., ${ }^{6}$ reported that the patients with hypothyroidism had a longer recovery time and reduced amplitude of stapedial muscle. Musiek \& Gollegly, Knipper \& Santos et al., ${ }^{8,9}$ reported that approximately $10-55 \%$ of all people with hypothyroidism have some hearing loss and the site of lesion is cochlea and central auditory pathways. ${ }^{10}$ Khechinaschvili et al. ${ }^{11}$ investigated 50 patients with hypo-function of thyroid gland and found that auditory system was affected secondary to hypothyroidism. A recent study by Karlos et al. investigated 30 hypothyroidism patients and reported bilateral mild to moderate sensorineural hearing loss. TEOAEs were absent and increased latency of waves I, III and V were found in ABR. They concluded that patients with hypothyroidism had higher audiometric thresholds, increase in interpeak latency between waves in the ABR and absence or reduction in TEOAEs amplitudes. ${ }^{12}$ Thornton AR and Jarvis SJ in a comparative study of hearing profile in hypothyroidism and hyperthyroidism in 35 patients, reported that 14 patients had same audiometric configuration at four frequencies average and 21 patients had hearing threshold above $25 \mathrm{~dB}$ and found that there was no significant difference between hyperthyroidism and control group's auditory profile. But they concluded that there was a significant involvement of retrocochlear auditory system in hypothyroidism patients. ${ }^{13}$ 
Hyperthyroidism is an autoimmune disorder marked by hyperactivity of the thyroid gland. Hyperthyroidism can be seen in Grave's disease, Thyrotoxicosis, Toxic Diffuse Goiter, Toxic Nodular Goiter, Plummer's Disease, Basedow's Disease etc. Bruschini et al., ${ }^{6}$ reported that in patients with hyperthyroidism neither the threshold nor the latency, the rise time, the recovery time and maximal amplitude of the acoustic reflex were different from normal subjects. Dokianakis et al., ${ }^{14}$ evaluated 23 hyperthyroidism patients and found that 12 patients had definite mild-moderate sensorineural hearing impairment. They also reported mixed hearing loss of mild to moderate degree in 4 patients. However, Thornton AR and Jarvis SJ did a comparative study of hearing profile in hypothyroidism and hyperthyroidism in 35 patients who reported that there was no significant difference between hyperthyroidism and control groups auditory profile. ${ }^{10}$

There is no agreement between the results of various studies in the literature pertaining to audiological findings of hypo and hyperthyroidism. Hence, the present study was conducted with the aim to assess audiological profile in hypo and hyperthyroidism and compare the findings with normal controls. The other aim was to compare between the two study groups.

\section{Materials and methods}

The study sample population comprised of 42 subjects (both males and females) diagnosed with hypothyroidism (Group 1: 22 subjects) and hyperthyroidism (Group 2: 20 subjects), in the age range of 18 to 45years. Control group (CG) consisted of 30 otologically and audiologically normal subjects of comparable age and sex. All hypothyroidism and hyperthyroidism cases were assessed after a confirmed diagnosis by an Endocrinologist based on thyroid function test (TFT) including T3, T4 and TSH (thyroid stimulating hormone) levels. The otoscopic examination was carried out by an ENT specialist. Patients without any external and middle ear were enrolled in study for audiological assessment. The subjects with history of thyroid hormone disorder involving other systems, otorrhea, ear trauma, ototoxicity, medical illness causing hearing loss, other genetic disorders and syndromes were excluded. The study was approved by the Institute Ethical Committee (IEC). The authors assert that all procedures contributing to this work comply with the ethical standards of the relevant national and institutional guidelines on human experimentation and with the Helsinki Declaration of 1975 , as revised in 2008 .

The comprehensive audiological test battery was carried out on all the subjects in the sound treated rooms which conform to the ANSI/ ISO standards for the maximum permissible noise levels. Puretone audiometry (PTA) between $250 \mathrm{~Hz}-8000 \mathrm{Hzwith}$ Telephonics TDH-39 supra-aural earphone, bone-conduction (BC) testing using Radio-ear $\mathrm{B}-71$ vibrator up to $4 \mathrm{KHz}$ and high frequency putretone audiometry
(HFPTA) between $10 \mathrm{kHz}-16 \mathrm{kHz}$ using SenheisserHDA200 earphone circum-aural headphone were conducted on a two channel clinical/diagnostic audiometer Madsen Orbiter 922 using bracketing method (modified Hughson and Westlake). Immitance audiometry (IA) and acoustic reflex testing (ART) was carried out with Maico MI-34 calibrated as per ANSI 1992 standards using $226 \mathrm{~Hz}$ probe tone. ART was measured for both ipsilateral and contralateral ears at $500 \mathrm{~Hz}, 1 \mathrm{kHz}, 2 \mathrm{kHz}$ and $4 \mathrm{kHz}$. Ipsilateral ART between 70-90 dB $\mathrm{HL}$ was considered as present and, between $95-110 \mathrm{~dB}$ as elevated. Contralateral ART between 80-100 dB HL was considered as present and between 105-120 dB HL as elevated. Both transient evoked otoacoustic emissions (TEOAEs) and distortion product otoacoustic emissions (DPOAEs) were measured using Intelligent Hearing System (IHS), USA. The amplitude and signal to noise ratio (SNR) at frequencies $1,000 \mathrm{~Hz}, 1,500 \mathrm{~Hz}, 2,000,3,000 \mathrm{~Hz}$ and $4,000 \mathrm{~Hz}$ were used to interpret the test results in TEOAEs. The SNR of $>3 \mathrm{~dB}$ at three consecutive frequencies, was considered as present. Neuroaudio (Russia) version 87 was used to measure ABR. The absolute latencies of wave I, III and V and the interpeak latencies of Wave I-III, III-V and I-V were studied.

\section{Results}

Statistical analysis for the comparison between three group viz. CG, hypothyroidism (group 1) and hyperthyroidism (group 2) was done through one way ANOVA. The age range of subjects was 18-45 years. The mean age of subjects in three groups were $31.43 \pm 6.67$ years, $31.18 \pm 7.30$ years and $30.61 \pm 7.58$ years respectively as shown in Table1. The mean values of T3, T4 and TSH hormone levels in group 1 were $4.64 \pm 14.08 \mathrm{ng} / \mathrm{ml}, 6.85 \pm 3.73 \mu \mathrm{g} / \mathrm{dl}$ and $39.73 \pm 65.22 \mu \mathrm{IU} / \mathrm{L}$ respectively. The mean values of T3, T4 and TSH hormone levels in group 2 were $62.48 \pm 61.80 \mathrm{ng} / \mathrm{dl}, 16.34 \pm 6.23 \mu \mathrm{g} / \mathrm{dl}$ and $1.78 \pm 2.84$ $\mu \mathrm{IU} / \mathrm{L}$ respectively.

\section{The results of various audiological assessments are en listed below}

Impedance Audiometry: The ear canal volume (ECV) was $1.32 \pm 0.27 \mathrm{ml}, 1.14 \pm 0.39 \mathrm{ml}$ and $1.12 \pm 0.34 \mathrm{ml}$ in $\mathrm{CG}$, groupl, and 2 respectively whereas it was $1.23 \pm 0.22 \mathrm{ml}, 1.25 \pm 0.35 \mathrm{ml}$ and $1.12 \pm 0.28 \mathrm{ml}$ respectively in CG, group 1 , and 2 in left ear. The static compliance (SC) in CG, group 1 and 2 was $0.83 \pm 0.34 \mathrm{ml}, 0.75 \pm 0.44 \mathrm{ml}$ and $0.60 \pm 0.38 \mathrm{ml}$ respectively and in left ear the $\mathrm{SC}$ was $0.96 \pm 0.48 \mathrm{ml}$, $0.85 \pm 0.57 \mathrm{ml}$ and $0.91 \pm 0.52 \mathrm{ml}$ respectively. The middle ear pressure (MEP) in CG, group1 and 2 was $7.73 \pm 14.33 \mathrm{daPa}, 1.68 \pm 10.67 \mathrm{daPa}$ and $-4.46 \pm 15.53 \mathrm{daPa}$ respectively in right ear and $5 \pm 18.47 \mathrm{daPa}$, $1.77 \pm 28.96 \mathrm{daPa}$ and $5.31 \pm 15.47 \mathrm{daPa}$ in left ear respectively. Statistically, there was no significant $(p>0.05)$ difference in ECV, SC and MEP among three groups.

Table I Comparison of PTAI, PTA2 (Air Conduction) and Bone Conduction among CG, GroupI and 2

\begin{tabular}{|c|c|c|c|c|c|c|c|c|}
\hline \multirow[t]{2}{*}{ Test } & \multirow[t]{2}{*}{ Ear } & \multicolumn{2}{|c|}{ CG $(n=30)$} & \multicolumn{2}{|c|}{ Group I $(n=22)$} & \multicolumn{2}{|c|}{ Group $2(n=20)$} & \multirow[t]{2}{*}{ F-ratio } \\
\hline & & Mean & SD & Mean & SD & Mean & SD & \\
\hline \multirow[t]{2}{*}{ PTAI (dB) } & Right & 11.22 & 3.21 & $18.03 * *$ & 2.8 & $16.91 * *$ & 3.47 & $31.065 * *$ \\
\hline & Left & 10 & 3 & $17.88^{* *}$ & 3.18 & $17.25 * *$ & 4.5 & $43.019 * *$ \\
\hline \multirow[t]{2}{*}{ PTA2(dB) } & Right & 28.45 & 6.5 & $57.32^{* *}$ & 13.5 & $36.92 * *$ & 12.66 & $|9.19| * *$ \\
\hline & Left & 28.58 & 8.32 & $54.26 * *$ & 13.46 & $37.26 * *$ & 12.46 & $12.10 \mid * *$ \\
\hline \multicolumn{2}{|c|}{ Bone Conduction(dB) } & 7.16 & 3.4 & $14.70^{* *}$ & 3.16 & $12.06 * *$ & 4.02 & $43.57^{* *}$ \\
\hline
\end{tabular}

$* *$ p-value $<0.0$ I =highly significant 
Table 2 Comparison of ABR among CG, GroupI and 2

\begin{tabular}{|c|c|c|c|c|c|c|c|c|}
\hline \multirow[t]{2}{*}{ Wave } & \multirow[t]{2}{*}{ Ear } & \multicolumn{2}{|c|}{ CG $(n=30)$} & \multicolumn{2}{|c|}{ Group I $(n=22)$} & \multicolumn{2}{|c|}{ Group $2(n=20)$} & \multirow[t]{2}{*}{ F-ratio } \\
\hline & & Mean & SD & Mean & SD & Mean & SD & \\
\hline \multirow[t]{2}{*}{1} & Right & 1.291 & 0.057 & 1.42 & 0.066 & 1.378 & 0.118 & $9.604^{*}$ \\
\hline & Left & 1.322 & 0.091 & 1.44 & 0.095 & 1.396 & 0.091 & $3.458^{*}$ \\
\hline \multirow[t]{2}{*}{ III } & Right & 3.37 & 0.118 & 3.509 & 0.144 & 3.487 & 0.196 & $3.862^{*}$ \\
\hline & Left & 3.4 & 0.148 & 3.553 & 0.114 & 3.531 & 0.198 & $4.385^{*}$ \\
\hline \multirow[t]{2}{*}{ V } & Right & 5.155 & 0.107 & 5.361 & 0.198 & 5.273 & 0.214 & $3.305^{*}$ \\
\hline & Left & 5.206 & 0.153 & 5.321 & 0.225 & 5.34 & 0.23 & $0.8 \mathrm{I} 5 \mathrm{~ns}$ \\
\hline \multirow[t]{2}{*}{ I-V } & Right & 3.862 & 0.116 & 3.896 & 0.199 & 3.892 & 0.18 & $0.079 n s$ \\
\hline & Left & 3.884 & 0.147 & 3.876 & 0.252 & 3.919 & 0.169 & $0.944 n s$ \\
\hline \multirow[t]{2}{*}{ I-III } & Right & 2.078 & 0.111 & 2.047 & 0.166 & 2.108 & 0.163 & $0.286 \mathrm{~ns}$ \\
\hline & Left & 2.078 & 0.138 & 2.109 & 0.122 & 2.112 & 0.159 & $0.663 \mathrm{~ns}$ \\
\hline \multirow[t]{2}{*}{ III-V } & Right & 1.785 & 0.131 & 1.863 & 0.203 & 1.786 & 0.188 & $0.243 \mathrm{~ns}$ \\
\hline & Left & 1.806 & 0.145 & 1.767 & 0.179 & 1.808 & 0.172 & $\mathrm{I} .53 \mathrm{Ins}$ \\
\hline
\end{tabular}

*Significant $(p<0.05)$; ns= not significant $(p>0.05)$

Acoustic reflex Testing (ART): Ipsilateral Reflexes: Acoustic reflex in right ear was present in $93.3 \%$ of subjects in CG, $9.1 \%$ of subjects in group 1 and $15 \%$ of subjects in group 2 however, elevated in $6.7 \%$, $86.36 \%$ and $85 \%$ of subjects in CG, group 1 and 2 respectively. Whereas in left ear, the acoustic reflex was present in $93.3 \%, 18.8 \%$ and $10 \%$ of CG, group 1 and 2 respectively whereas elevated in $6.7 \%$ of subjects in CG, $81.82 \%$ of subjects in group 1 and $90 \%$ of subjects in group2 as shown in Graph1. On applying Pearson's Chi-square test, highly significant $(\mathrm{p}<0.01)$ differences were observed among three groups.

The contralateral acoustic reflex in right ear was present in $96.6 \%$, $13.63 \%$ and $15 \%$ of the subjects in CG, group1 and 2 respectively whereas, elevated in $3.33 \%, 86.36 \%$ and $85 \%$ of the subjects in $\mathrm{CG}$, group1 and 2 respectively. However, in left ear, the acoustic reflex was present in $90 \%$ of the subjects in CG, $13.63 \%$ of the subjects in group 1 and $15 \%$ of the subjects in group 2 whereas elevated in $10 \%, 86.36 \%$ and $85 \%$ of subjects in CG, group 1 and 2 respectively as shown in Graph2. Statistically, there was a significant difference $(p<0.01)$ among three groups in both ears

Otoacoustic Emission (OAE): TEOAEs were found to be present in $90 \%, 50 \%$ and $69.2 \%$ of the subjects in CG, group 1 and 2, whereas absent in $10 \%, 50 \%$ and $30.8 \%$ of the subjects in CG, group 1 and 2 respectively in right ear. In left ear, TEOAEs were present in $93.3 \%$ of the subjects in CG, $40.9 \%$ of the subjects in group 1 and $80 \%$ of the subjects in group 2 whereas absent in $6.7 \%$ of the subjects in CG, and $59.1 \%$ of the subjects in group 1 and $20 \%$ of the subjects in group 2 as shown in Graph2. There was a highly significant difference $(\mathrm{p}<0.01)$ among three groups in both left and right ears.

Results revealed that DPAOEs were present in $93.3 \%$ of the subjects in CG, $68.2 \%$ of the subjects in group 1 , and $85 \%$ of the subjects in group2 however absent in $6.7 \%$ of the subjects in CG, $31.8 \%$ of the subjects in group 1 and $15 \%$ of the subjects in group 2 in right ear. In left ear DPOAEs were present in $93.3 \%$ of the subjects in CG, $72.7 \%$ of the subjects in group 1 and $80 \%$ of the subjects in group 2 whereas absent in $6.7 \%, 27.3 \%$ and $20 \%$ of the subjects in $\mathrm{CG}$, group1 and 2 respectively as shown in Graph2. On applying
Chi-square test, no significant ( $p>0.05$ ) difference was found between three groups in both right and left ear.

Pure Tone Audiometry(PTA): PTA1 (average threshold at frequencies $500 \mathrm{~Hz}, 1000 \mathrm{~Hz}$ and $2000 \mathrm{~Hz}$ ) was calculated. The mean PTA1 in right ear of CG, group1 and 2 was $11.2 \pm 3.21 \mathrm{~dB}, 18.03 \pm 2.8$ $\mathrm{dB}$ and $16.91 \pm 4.47 \mathrm{~dB}$ respectively. The mean PTA1 for left ear was $10 \pm 3.0 \mathrm{~dB}$ of $\mathrm{CG}, 17.88 \pm 3.18 \mathrm{~dB}$ of group1 and $17.25 \pm 4.5$ $\mathrm{dB}$ of group2 as shown in Table2. Statistically, significant $(\mathrm{p}<0.01)$ difference between the three groups was observed for PTA1 in both right and left ear. On post hoc multiple comparison Bonferroni test, it was found that there was also a statistically significant $(p<0.01)$ difference between the CG and group1; CG and group2 as well as between group1 and group2 in both right and left ear.

Extended High Frequency (HF) audiometry: PTA2 (average of $10000 \mathrm{~Hz}, 12000 \mathrm{~Hz}$ and $14000 \mathrm{~Hz}$ ) was analyzed for both ears in all groups. The mean of PTA2 of right ear was $28.45 \pm 6.50 \mathrm{~dB}$ in $\mathrm{CG}$, $57.32 \pm 13.50 \mathrm{~dB}$ in group1 and $39.92 \pm 12.66 \mathrm{~dB}$ in group2 whereas $28.58 \pm 8.32 \mathrm{~dB}$ in $\mathrm{CG}, 54.26 \pm 13.46 \mathrm{~dB}$ in group 1 and $37.26 \pm 12.46$ $\mathrm{dB}$ in group2 of left ear respectively as shown in Table 2 . Statistically significant $(\mathrm{p}<0.01)$ differences were found among three groups. On pair wise comparison statistically significant $(p<0.01)$ difference was observed between CG and 1; and between CG and group2. Statistically significant difference $(\mathrm{p}<0.05)$ was also observed between the group 1 and 2 in both ears as shown in Table 1

Bone Conduction (BC) Threshold (Table 1): The mean of PTA1 bone conduction was $7.16 \pm 3.4 \mathrm{~dB}$ in $\mathrm{CG}, 14.6 \pm 3.15 \mathrm{~dB}$ in group1, and $12.06 \pm 4.02 \mathrm{~dB}$ in group2. The pair wise comparison between the two groups revealed statistically highly significant $(\mathrm{p}<0.01)$ difference between the CG and group1; CG and group2. However, no significant difference $(\mathrm{p}>0.05)$ was seen between group1 and group2 as shown in Table 1.

Degree of hearing loss: The degree of hearing loss was categorized on the classification system recommended by American Speech and Hearing Association (ASHA).

Right Ear: In CG, $96.7 \%$ of the subjects had normal hearing and $3.3 \%$ had minimal hearing loss. However, in group $1,22.7 \%$ of the 
subjects had normal hearing and $77.3 \%$ of the subjects had minimal hearing loss whereas $15 \%$ of the subjects had normal hearing and $85 \%$ of the subjects had minimal hearing loss in group2 as shown in Graph3. Results revealed highly significant $(\mathrm{p}<0.01)$ difference between three groups viz.CG, group $1 \& 2$.

Left Ear: In CG, all the subjects had normal hearing. However, in group $1,22.7 \%$ had normal and $77.2 \%$ of the subjects had minimal hearing loss whereas $25 \%$ had normal and $75 \%$ of the subjects had minimal hearing loss in group2 as shown in Graph3. Results showed statistically highly significant difference $(p<0.01)$ between three group viz. CG, group 1 and 2 . Therefore, the results showed that the minimal hearing loss was more prevalent in group1 and group2.

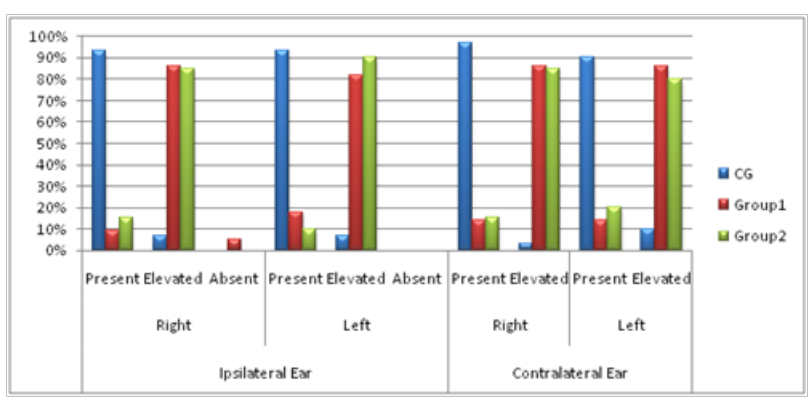

Graph I Acoustic Reflex Threshold (ART).

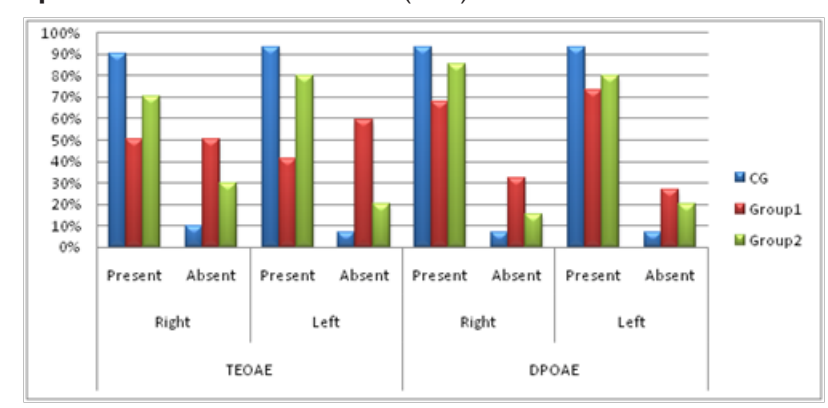

Graph 2 Otoacoustic Emission (OAE).

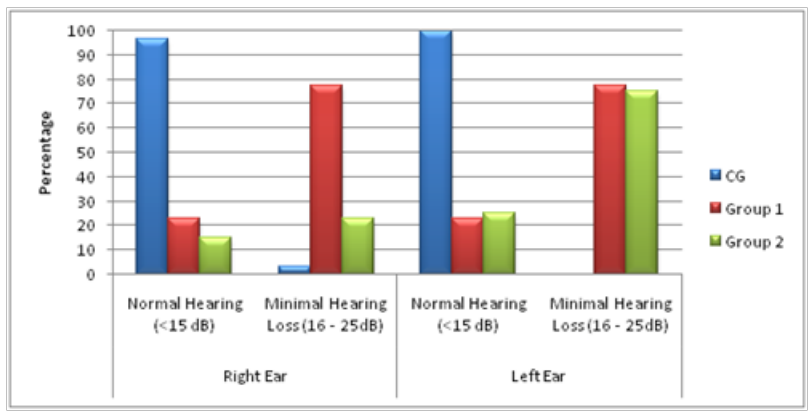

Graph 3 Degree of Hearing Loss.

Auditory Brainstem Evoked Responses (ABR): Latencies and interpeak latency for Wave I, III and V were compared among three groups using one way ANOVA. The results revealed a significant difference in latency for wave I and III for both ears and Wave V for right ear. No significant difference was observed for absolute latency for wave $\mathrm{V}$ in left ear. Results also revealed no significant difference between three groups for inter-peak latency I-V, I-III and III-V in either ear as shown in Table 2.

On applying the Post hoc multiple comparisons Bonferroni Test, results revealed significant difference between $C G$ and group1 for absolute latency of wave-I, III and V in right ear and absolute latency of I and III wave. Significant difference was observed between CG and group2 for the latency of wave I in right ear and for the latency of wave III in left ear. Significant difference was observed between group 1 and 2 for the absolute latency of wave I and V in right ear only. However, there was no significant difference between CG and 2 and group1 and 2 for inter-peak latency in either ear.

\section{Discussion}

An association between hearing and thyroid function has long been reported in various studies. Still there are several unclear issues related to the true incidence and degree of hearing loss. Thyroid hormones play multiple physiologic effects in our body system. ${ }^{15}$ In addition, thyroid hormone is important for normal development of the auditory system. ${ }^{5}$ Regardless of knowing that thyroid hormones are involved in structure and physiological development of the inner ear, the molecular basis for the permanent nature of hypothyroidisminduced hearing loss is still not clear.

In the present study, the detailed audiological assessment in patients having acquired hypothyroidism and hyperthyroidism was conducted to investigate the degree and nature of hearing loss. Subjects with hypothyroidism were characterized by normal serum level of free T4 and increased level of TSH in blood. Subjects with hyperthyroidism had normal serum level of TSH and increased level of T3. In the present study all subjects who had external or middle ear pathology were excluded and the results revealed no reduction in MEP and SC in impedance audiometry i.e, all subjects had SC and MEP within normal range. However, Dokianakis et al. ${ }^{14}$ observed in their study that out of 23 hypothyroid patients, four subjects had reduced middle ear pressure (less than-100 daPa) and compliance in which three had $300 \mathrm{~mm}$ of $\mathrm{H}_{2} \mathrm{O}$ and one had a seromucotympanum before treatment which improved after treatment. Their study included subjects with middle ear pathology which could be the reason for discrepancy between present studies. Bhatia et al., ${ }^{16}$ and Rubenstein et al., ${ }^{17}$ also reported the conductive component in impedance audiometry. Bhatia et al., ${ }^{16}$ excluded all those subjects who had known cause of hearing loss and in impedance audiometry out of 72 patients, $8.3 \%$ of the patients had middle ear pathology. ${ }^{16,17}$ Other previous studies have also shown the similar observations. ${ }^{18,19}$ ARTs in three groups, viz. control, hypothyroidism and hyperthyroidism subjects were measured and results revealed highly significant difference $(p<0.01)$ in ARTs of CG as compared to group1 and group2 in both ears ipsilaterally as well as contralaterally. The results showed that the mean ARTs for ipsilateral as well as contralateral ear of group1 and group2 subjects were elevated as compared to $\mathrm{CG}$ at all tested frequencies in both ears. However, results revealed no significant difference $(p>0.05)$ between two study groups, viz. group1 and group 2 in both ears for ipsilateral as well as contralateral reflexes. This is in agreement with Bruschini et al. ${ }^{6}$ did not find significant difference between hypothyroid and hyperthyroid patients in ARTs characteristics. ${ }^{6}$ In the present study, TEOAEs were present in $50 \%$ and $40.9 \%$ of the subjects in right and left ear respectively in group 1 however, absent in $50 \%$ of the subjects in right ear and $59.1 \%$ of the subjects in left ear. TEOAEs were present in $69.2 \%$ and $76.9 \%$ of the subjects in right and left ear respectively in group 2 i.e, hyperthyroidism. This is in agreement with various previous studies reporting reduced SNR in hypothyroidism patients. Khechinaschvili et al., ${ }^{11}$ studied 50 subjects with hypothyroidism and found that $52 \%$ of the subjects had absent TEOAEs and stated that it is due to the decreased pathophysiology and reduced activity of cells in the organ of Corti of the cochlea., ${ }^{11}$ In the present study, DPOAEs were present in $84.6 \%$ of the subjects whereas, absent in $15.4 \%$ in both ears in hyperthyroidism. Karlos et 
al. stated that the pathophysiology behind the reduction in SNR in OAE may be due to hypotrophy of cells present in striavascularis and organ of Corti. ${ }^{12}$ Results showed that $77.3 \%$ of subjects had minimal hearing loss in both ears in group 1 . This is in agreement with Khechinaschvili et al., ${ }^{11}$ who reported hearing loss in $74 \%$ of subjects had hearing loss and stated that the pathophysiological changes in hearing may involve many auditory pathway sites including endocochlear, retrocochlear and central pathways. ${ }^{11}$ However, Thornton and Jarvis ${ }^{10}$ in a comparative study between hypothyroid and hyperthyroid found that the average threshold was above $25 \mathrm{~dB}$ in hypothyroidism however there was no significant difference between control and hyperthyroidism and they hypothesized that the significant involvement may be due to alteration in hormone level which leads to change in metabolism and pathophysiological changes of cells of the system including retrocochlear auditory system. Results also revealed highly significant difference among all three groups for high frequency viz. $10000 \mathrm{~Hz}$ and $12000 \mathrm{~Hz}$ and $14000 \mathrm{~Hz}$ frequency in both ears. No study could be found in the literature reporting comparison between hypothyroidism and hyperthyroidism on extended high frequency hearing sensitivity. However, Berker et al., ${ }^{13}$ studied 22 subjects with Grave's disease had also reported sensorineural hearing loss especially at higher frequency in the majority of the subjects., ${ }^{13}$ The auditory brainstem evoked response audiometry showed significant difference for absolute latency of wave I and III in both ears and wave V for right ear. Karlos \& Chandrasekhar et al., ${ }^{12,20}$ have also reported the increased absolute latencies of waves that might be due to decreased metabolism of tissues which may result in slow conduction of periphery and poor recruitment of neuronal pool of the generators of the waves in brainstem. Results showed there was no significant difference $(p>0.05)$ between CG and group2 (hyperthyroidism). In concordance, Thornton AR, Jarvis SG also found no significant ( $>0.05)$ difference for absolute as well as inter-peak latency of waves in hyperthyroidism as compared to control. They also stated that the retrocochlear involvement may be associated with body temperature of patients in hypothyroidism so it may affect absolute latency \& interpeak latencies of waves in ABR. ${ }^{10}$

\section{Conclusion}

In both hypothyroidism and hyperthyroidism subjects, a minimal degree of hearing loss is found in about $80 \%$ of subjects including the extended high frequency and it mainly affects the cochlea due to affected metabolism and not the external and middle ear. The acoustic reflex in response to loud sounds was also elevated for minimal hearing loss in both hypothyroidism and hyperthyroidism. The absolute latency of waves in ABR was significantly delayed in hypothyroidism as compared to the control and hyperthyroidism. However, in a multiple comparison no significant difference was observed between control and hyperthyroidism, suggested the involvement of brainstem also in the hypothyroidism subjects. So, among the hypothyroidism and hyperthyroidism, the auditory impairment is in greater extent in hypothyroidism involving both cochlear and retrocochlear auditory system of the subjects as compared to hyperthyroidism.

\section{Acknowledgments}

None.

\section{Conflicts of interest}

Author declares there are no conflicts of interest.

\section{Funding}

None.

\section{References}

1. Bernal J. Thyroid hormones and brain development. Vitm Horm. 2005;71:95-122.

2. Unnikrishnan AG, Menon UV. Thyroid disorder in India: An epidemiological perspective. Indian J Endocrinol Metab. 2011;15(Suppl 2):S78-S81

3. Deol MS. Congenital deafness and hypothyroidism. Lancet. 1973;2(7820):105-106.

4. Uziel A. Periods of sensitivity to thyroid hormone during the development of the organ of Corti. Acta Otolaryngol Suppl. 1986;429:23-27.

5. Sohmer H, Freeman S. The importance of thyroid hormone for auditory development in the fetus and neonate. Audiol Neurootol. 1996;1(3):137-147.

6. Bruschini P, Sellari FS, Bartalena L, et al. Acoustic reflex characteristics in hypo- and hyperthyroid patients. Audiology. 1984;23(1):38-45.

7. Musiek FE, Gollegely MK. ABR in eighth nerve and low brainstem lesions. In: Jacobsen (Edr,). The auditory brainstem response. The College-Hill Press, Sand Diego, USA,. 1985. p. 181- 202.

8. Knipper M, Zinn C, Maier H, et al. Thyroid hormone deficiency before the onset of hearing causes irreversible damage to peripheral and central auditory systems. J Neurophysiol. 2000;83(5):3101-3112.

9. Santos KT, Dias NH, Mazeto GM, et al. Audiologic evaluation in patients with acquired hypothyroidism. Braz J Otorhinolaryngol. 2010;76(4):478-484.

10. Thornton AR, Jarvis SJ. Auditory brainstem response findings in hypothyroid and hyperthyroid disease. Clin Neurophysiol. 2008;119(4):786-790.

11. Khechinaschvili S, Metreveli D, Svanidze N, et al. The hearing system under thyroid hypofunction. Georgian Med News. 2007;(144):30-33.

12. Santos KT, Dias NH, Mazeto GM, et al. Audiologic evaluation in patients with acquired hypothyroidism. Braz J Otorhinolaryngol. 2010;76(4):478-484.

13. Berker D, Karabulut H, Isik S, et al. Evaluation of hearing loss in patients with Graves‘ disease. Endocrine. 2012;41(1):116-121.

14. Dokianakis G, Ferekidis E, Pantazopoulos P. [Hearing loss and hyperthyroidism (author's) transl) ]. Arch Otorhinolaryngol. 1978;219(2):351-353.

15. Salvatore D, Davies TF, Schlumberger J, et al. Thyroid physiology and diagnostic evaluation of patients with thyroid disorders. In: Melmed S, Polonsky KS, Larsen PR, Kronenberg HM (eds) Williams Textbook of endocrinology, 12th edition. Elsevier Saunders, Philadelphia. 2011. P.327-361.

16. Bhatia PL, Gupta OP, Agrawa MK, et al. Audiological and vestibular function tests in hypothyroidism. Laryngoscope. 1977;87(12):2082-2089.

17. Rubenstein M, Rubenstein C, Theodor R. Hearing dysfunction associated with congenital sporadic hypothyroidism. Ann Otol Rhinol Laryngol. 1974;83(6):814-819.

18. Hilger JA. Otolaryngologic aspects of hypometabolism. Annals of OtolRhinol Laryngol. 1956;65(2):395-413.

19. Howarth AF Lloyd HE. Perceptive Deafness in Hypothyroidism. $\mathrm{Br}$ Med J. 1956;1(4964):431-433.

20. Chanderashekhar M, Kowsalya V, Vijyalakshmi B. Electrophysiological changes on brainstem auditory evoked potentials in hypothyroid patients. J Pharmacy Research. 2011;4(8):2856-2859. 